\title{
Survey of Radio Navigation Systems
}

\author{
Przemysław Gilski and Jacek Stefański
}

\begin{abstract}
At present, there is a growing demand for radio navigation systems, ranging from pedestrian navigation to consumer behavior analysis. These systems have been successfully used in many applications and have become very popular in recent years. In this paper we present a review of selected wireless positioning solutions operating in both indoor and outdoor environments. We describe different positioning techniques, methods, systems, as well as information processing mechanisms.
\end{abstract}

Keywords-radio navigation, radio communication, satellite navigation systems, radiofrequency identification

\section{INTRODUCTION}

$\mathbf{S}$ ERVICES for which it is crucial to know the position of a user or a mobile terminal are referred to as LBS (LocationBased Services). LBS are services for providing information that has been created, compiled, selected and filtered taking into consideration the current location of a person or device. They can appear in conjunction with conventional services like telephony and related value-added features. The attractiveness of LBS results from the fact that participants do not have to enter location information manually, they are automatically pinpointed and tracked.

Nowadays, positioning systems are used most often for navigation purposes, especially to shorten delivery time, also to detect the presence of people, monitor user traffic and determine movement patterns. Currently, more and more malls and shopping centers analyze customer behavior in order to optimize store infrastructure. Furthermore, knowledge about the location of a handheld device, combined with a user profile, can significantly improve network planning, traffic analysis and radio resource management.

\section{FUNDAMENTALS OF POSITIONING}

A very important subservice needed for building a LBS is the so-called LCS (LoCation Service). LBS uses location data in order to compile, filter and select information or provide other added value to the user depending on location data, whereas LCS is primarily concerned with the mere delivery of location data. Compared to position fixes, which only contain a position and perhaps the target's identity or the type of positioning method used, location data contains additional high-level information that is more appropriate for processing by a LBS application [1][2].

Positioning methods can be divided according to the means in which the signal received by a handheld device is measured. In a wireless communication system the location of a mobile

P. Gilski and J. Stefański are with the Department of Radio Communication Systems and Networks, Faculty of Electronics, Telecommunications and Informatics, Gdansk University of Technology, Gabriela Narutowicza 11/12, 80-233 Gdansk, Poland (e-mail: pgilski@eti.pg.gda.pl; jstef@eti.pg.gda.pl). terminal is determined either by the terminal itself (terminalbased approach) or calculated by the network using signal metrics from the UE (User Equipment) (network-based approach). There are of course hybrid methods involving measurements and position estimation as a combination of both network and handheld device (non-conventional approach) [3]-[5].

\section{A. Network-Based Approach}

In case of network-based methods the position of a mobile terminal is obtained by performing measurements at the BTS (Base Transceiver Station). In most cases all data collected by the BTS is then sent to a dedicated location server such as the MPC (Mobile Position Center) or GMLC (Gateway Mobile Location Center) used in 2G (Second Generation) and 3G (Third Generation) cellular networks, where it is processed. The majority of equipment vendors has its own positioning solution, generally referred to as the LMU (Location Measurement Unit), which observes the transmission of data bursts either from different base stations or from terminals in its surrounding area in order to perform timing measurements.

Timing methods such as ToA (Time of Arrival) and TDoA (Time Difference of Arrival) require at least three BTS in order to determine the position of a handheld device.

In ToA method the distance between the terminal and a particular BTS is directly proportional to the propagation time. This method requires precise synchronization of both transmitter and receiver, also a time stamp provided by the transmitter in order to calculate the time difference and distance.

The TDoA method is very much alike, however instead of observing the time of arrival of a signal at a BTS it uses the time difference at which the signal arrives at numerous base stations. The intersection of hyperbolic curves represents the estimated location, which is shown in Fig. 1.

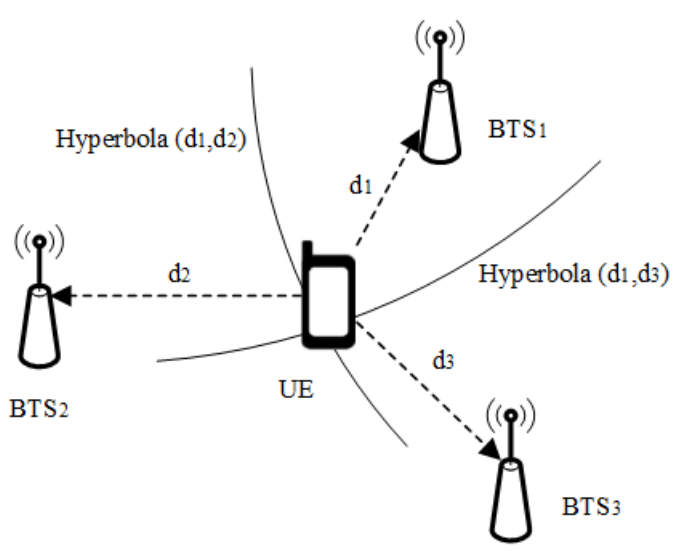

Fig. 1. TDoA positioning method 
The AoA (Angle of Arrival) method determines the location of a mobile terminal using directionally-sensitive antennas. This requires a network with complex-phased array base stations and an adaptive real-time processing unit in order to provide full beam-steering capabilities. The position of a terminal is estimated by the intersection of two or more bearings, as shown in Fig. 2.

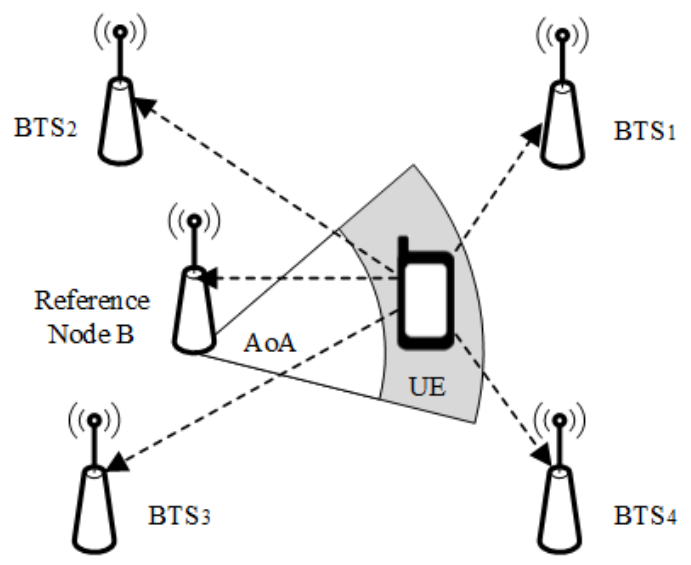

Fig. 2. AoA positioning method

Unfortunately, the accuracy of this method can be lowered due to multipath propagation, in a case when the strongest arriving signal may not have come along the bearing line, but a deceptive reflection from a building or moving object. Additionally, spatial resolution declines with increasing distance.

Cell-ID (Cell IDentification) is considered to be the simplest network-based positioning method. In a cellular network the whole area of operation is divided into hexagonal cells, each with a unique identification code. When a mobile terminal moves within this honeycomb structure, it connects with a BTS that provides the strongest signal. However, this method is not very accurate, because the estimated position is limited only to the area of the serving cell. In case of sectorized antennas, the potential position can be narrowed depending on the angle of sectors, as shown in Fig. 3.
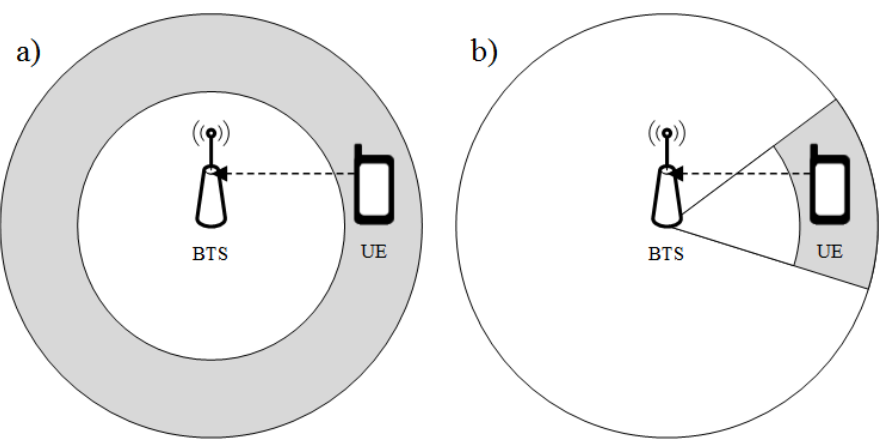

Fig. 3. Cell-ID positioning method a) omnidirectional antennas, b) sectorized antennas

For urban areas the size of a cell can vary from 1 to $3 \mathrm{~km}$, whereas in suburban or rural areas ranges from 10 up to $30 \mathrm{~km}$.

\section{B. Terminal-Based Approach}

There is a group of positioning methods which can be performed by the terminal itself. However, they have measurement limitations, for instance caused by the lack of an adaptive antenna array. That is why they rely mainly on signal measurements such as signal strength or a time-based approach.

Like ToA, OTD (Observed Time Difference) methods such as E-OTD (Enhanced OTD) and OTDoA (Observed Time Difference of Arrival) use signals sent from at least three BTS.

The E-OTD method used in GSM (Global System for Mobile communications) is based on LMU units, which contain an accurate time source and compute clock differences between numerous base stations. Fig. 4. represents the E-OTD method.

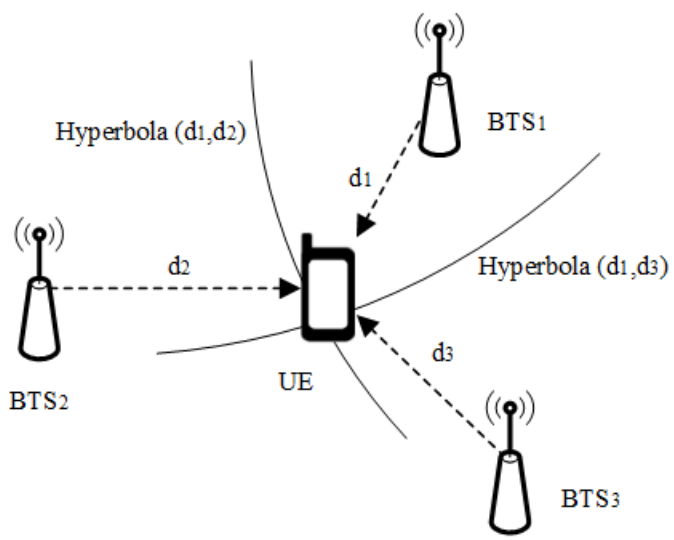

Fig. 4. E-OTD positioning method

When a mobile terminal receives at least three signals, the time differences can be calculated. The location is then estimated as the intersection of hyperbolic curves.

Its equivalent in UMTS (Universal Mobile Telecommunications System), called OTDoA, is based on the same principles. However, improvements had to be made due to the near-far effect. In this case idle periods are inserted into Node B transmissions in order to maximize the probability of receiving distant Node $\mathrm{B}$ pilot signals.

GPS (Global Positioning System) is so far the most accurate location technology. Nevertheless, it takes some time to obtain ones position after turning on a mobile terminal, which is called TTFF (Time To First Fix). Furthermore, the signal from the satellite is fairly weak due to the great distance. It is not possible to obtain a position indoors and even tall buildings or weather conditions can affect the signal. This system works best when LOS (Line Of Sight) conditions are provided to at least 4 out of 24 satellites. That is why an improvement had to be introduced.

A-GPS (Assisted GPS) uses not only satellites but also cellular networks to gain position. The SMLC (Serving Mobile Location Center) controls the entire positioning process, including allocation of resources, evaluation of timing measurements and calculation of position fixes. Fig. 5. illustrates the structure of the A-GPS system.

In this case the terminal can submit a request to the network for additional location data. Having information about the position of the mobile station the network notifies the terminal which satellites should be visible. The initial localization estimation can be performed by the terminal itself or calculated by the network. 


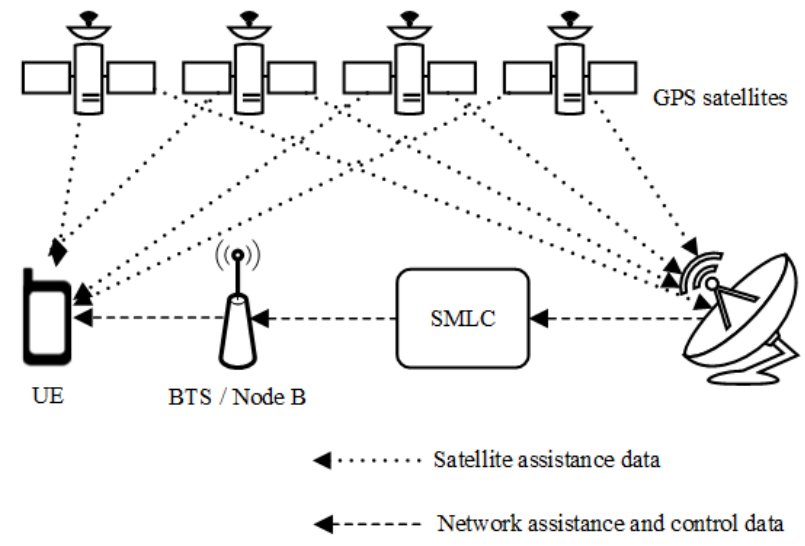

Fig. 5. A-GPS positioning method

The principle of RSS (Received Signal Strength) method is the fact, that each MS (Mobile Station) receives a unique set of signals from surrounding BSs (Base Stations). Due to the unstable propagation channel in the urban area RSS suffers from fluctuations caused by multipath propagation and user orientation. Fig. 6 describes the concept of RSS.

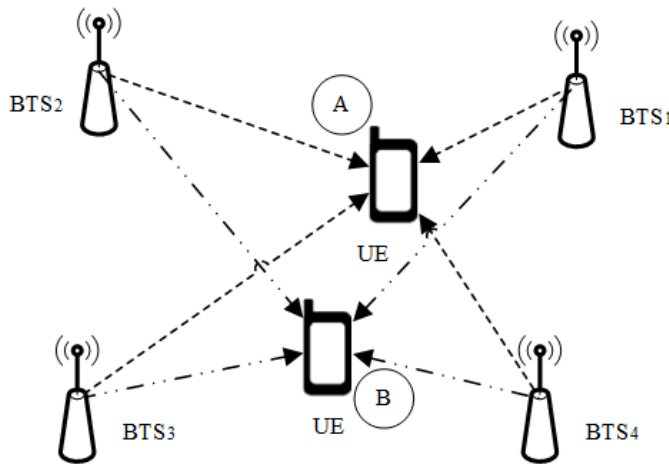

Fig. 6. RSS positioning method

However, in the indoor environment multipath propagation can significantly raise location performance, because it increases the rareness of the RSS fingerprint [6].

\section{Non-Conventional Approach}

Nowadays, there are some alternatives to the conventional positioning methods, based mainly on Bluetooth, WLAN (Wireless Local Area Network) and RFID (Radio Frequency IDentification). These novel methods involve calculations performed entirely by the terminal itself and also measurements carried out in cooperation with the network [7][8].

Bluetooth transceivers need to be installed in a desired area in such a way, to enable a mobile terminal to communicate with at least three devices in order to receive a position fix. The accuracy of this method is quite high since the coverage of a Bluetooth device achieves $100 \mathrm{~m}$ at most. Due to high costs and limited range this technology is mainly used in indoor scenarios.

WLAN positioning, based on RSS, is quite commonly used in urban areas where access points are available. In this case every operating AP (Access Point) transmits certain unique data, like its MAC (Media Access Control) address or SSID (Service Set IDentifier), in the form of beacons. It is quite likely that any mobile terminal can see more than one device at a particular point. All this data is stored in a vector, which can then act as a fingerprint for that position, and can be later used to estimate the location of the UE. The fingerprints are stored in a database, that has to be queried in order to receive the position. Of course, the more fingerprints stored in a database, the more precise localization [9]-[11].

RFID is an emerging technology used nowadays primarily for asset management, access control, textile identification and factory automation. It is based on radio signals exchanged between an RFID reader and tag.

The reader consists of an antenna, transceiver, power supply and an interface for connecting it to the server, e.g. via Ethernet, whereas a tag is simply a microchip. Tags can be divided into two groups: active and passive. Active tags are equipped with a power supply in the form of batteries, while passive extract the required energy from radio signals emitted by the reader. Active tags typically provide communication range of several meters, whereas passive assure a range of tens of centimeters. Furthermore, active tags contain more memory and are often equipped with additional sensors.

\section{Currently Available Solutions}

Designing systems and solutions for indoor positioning and navigation is a promising, yet complex task. It requires creating maps based on floor plans, choosing the most effective localization technology and algorithms, also deploying positioning devices. Most modern systems offer much more than simply localization with acceptable accuracy. They provide the user with additional context information in text, audio or video format and also collect statistical information about people movement.

\section{A. Smartmuseum}

The Smartmuseum project is an European recommendation system for museum visitors, supporting both indoor and outdoor excursions with positioning. Its mechanism is based on user search requests and profiles. Fig. 7 represents the architecture of Smartmuseum.

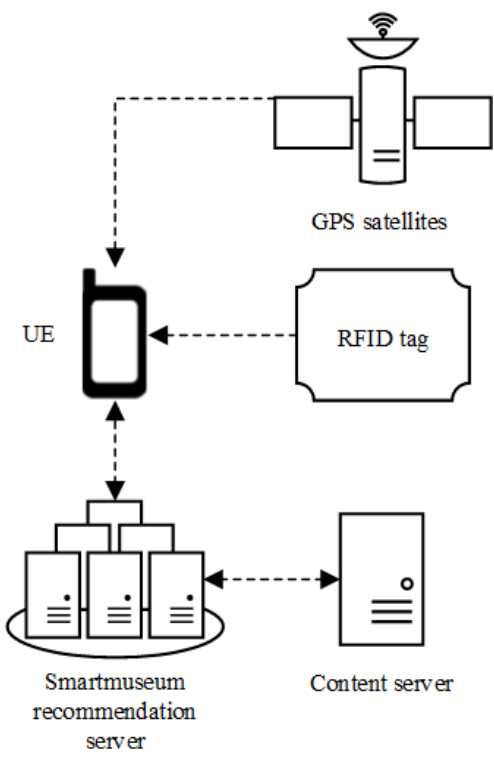

Fig. 7. Architecture of Smartmuseum 
Firstly, one should register in the system and provide information about his or her interests. Like a classic audio guide, the platform may provide the user with additional data about desired places or objects, using GPS for outdoor and RFID for indoor scenarios. Each RFID tag stores an URL (Uniform Resource Locator) web page address with information about the subject of cultural heritage. This service is currently available in Malta and Italy for Windows Mobile and Symbian OS (Operating System) [12].

\section{B. IWAY}

IWAY Indoor Positioning is a company focused on designing mobile navigation systems using different types of modules for different types of buildings according to their destination, e.g. supermarkets, convention centers, museums, hospitals, airports and offices. In case of a shopping center, a customer can simply download an application from the Internet in order to obtain information about discounts and special offers. In addition, the system provides the administration with an analysis of user movement and helps to evaluate the efficiency of the shop work and staff.

IWAY solutions include mobile and desktop modules for public facilities, staff and administration. The desktop part of the system is responsible for the analysis, statistical data processing and HR (Human Resources) management. Their technology is based on fingerprints, such as WiFi (Wireless Fidelity), GSM and 3G/4G (Fourth Generation) signals, with accuracy of 1 to $5 \mathrm{~m}$ [13].

\section{Walkbase}

This technology on the other hand has a different approach, because instead of displaying clients coordinates on the map it determines whether a user is located in a particular location or not with an accuracy of 3 to $5 \mathrm{~m}$. Walkbase uses a sensor that detects presence of customers with WiFi-enabled phones. It can monitor traffic outside and inside a mall, airport or other venue in order to find popular routes and movement patterns. As an analytics platform, Walkbase can monitor how many people came to a particular store, how it varied between locations and does the time spent convert to sales [14].

\section{Guardly}

Guardly is a company focused on designing LBS services for public safety purposes. These solutions utilize fingerprinting methods based on WLAN and Bluetooth signals. Fig. 8 describes the architecture of Guardly.

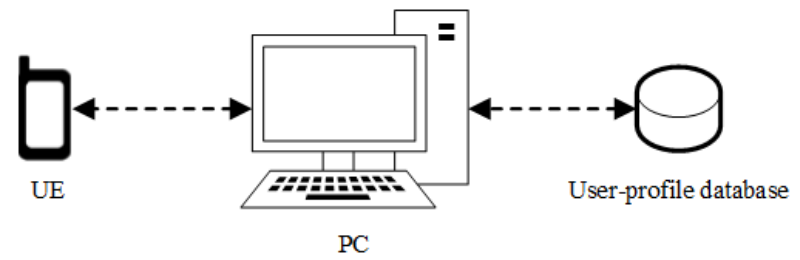

Fig. 8. Architecture of Guardly

The position of a mobile terminal is determined by a PC connected to a user-profile database. It is mandatory to create a unique profile for each user. When an alert message is issued, the system first determines the user's ID and then his or her position. Guardly offers comprehensive solutions for scientific and academic institutions (Safe Campus) as well as security agencies (Guardly Command) [15].

\section{E. Google Maps}

Google Maps is a service available for both mobile terminal and desktop PC (Personal Computer). In case of a PC this system utilizes only WiFi access points, with their MAC, SSID and RSS, whereas for mobile terminals it relies also on Cell-ID and GPS. Google Maps enables also to receive a position fix indoors through A-GPS. Fig. 9 represents the architecture of Google Maps.

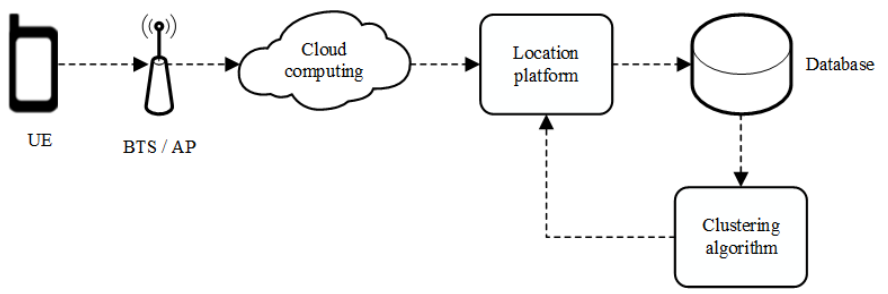

Fig. 9. Architecture of Google Maps

All collected data is then send to Google servers, where it is processed, and helps to build up a database for other services [16].

\section{F. Skyhook}

Skyhook Wireless is one of the main competitors of Google, which offers a SDK (Software Development Kit) for building location services (Skyhook Location SDK) and their own positioning systems including SpotRank and Skyhook Location Performance. The company uses hybrid location algorithms processing GPS, A-GPS, WiFi and cellular network signals, achieving accuracy of $10 \mathrm{~m}$ and a TTFF of just $1 \mathrm{~s}$. Skyhook utilizes a database of more than 700 million access points and base stations. It also enables to determine the density of people in indoor and outdoor environments [17].

\section{G. Qubulus}

This positioning platform specializes in solving navigation problems in indoor and outdoor environments using WiFi, 2G and $3 \mathrm{G}$ fingerprints. All collected data is then compressed into a special FPD file. The estimated position can be presented in the form of geo-coordinates and displayed on Google Maps and Bing Maps. Qubulus also provides the ability for consumers to write applications with the could API (Application Programming Interface) for Android OS [18].

\section{H. Nokia HAIP}

Nokia Research Center has developed a revolutionary HAIP (High Accuracy Indoor Positioning) technology based on AoA, using a modified version of BLE (Bluetooth Low Energy) for cost efficient and highly accurate indoor positioning.

This technology, based on directional positioning reference stations (locators) installed in a covered areas, does not require calibration and provides accuracy up to $0.3 \mathrm{~m}$. Fig. 10 represents the architecture of HAIP. 


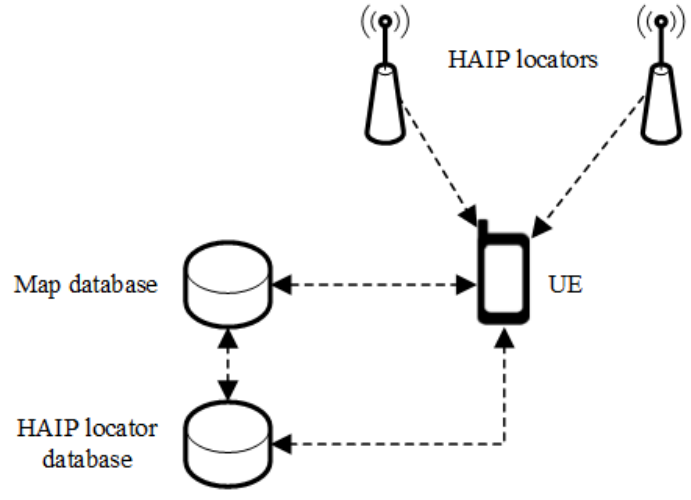

Fig. 10. Architecture of Nokia HAIP

In August 2012, Nokia co-founded the In-Location Alliance with 22 other companies. Currently, the consortium includes 51 members, e.g. Samsung, Sony, Huawei, Qualcomm, Intel, Cisco and Philips. Their main goal is to develop solutions offering high accuracy, mobility, usability and low power consumption in order to enhance consumer experience [19][20].

\section{Nokia HERE}

HERE is a company focused on developing maps of multistorey buildings, including shopping and conference centers, airports, museums and sport facilities. Until now the company has elaborated more than 9,000 large-scale objects, consisting of over 50,000 buildings in 69 countries around the world [21].

\section{J. Qualcomm IZat}

As a subsidiary company of Qualcomm Artheos, IZat is one of the main recipients of Nokia HERE. IZAT offers specialized LBS services, facilitating the functionality of users in a closed environment, e.g. navigation and information about discounts and special offers.

This technology is based on cellular, WLAN and sensor networks, as well as GPS satellite signals and cloud-aided solutions. It provides positioning accuracy of 3 to $7 \mathrm{~m}$ [22].

\section{K. Estimote}

Estimote is a new invention designed to introduce improvements in the retail sector. Specially designed beacons are attached to tables or walls. Fig. 11 demonstrates the architecture of Estimote.

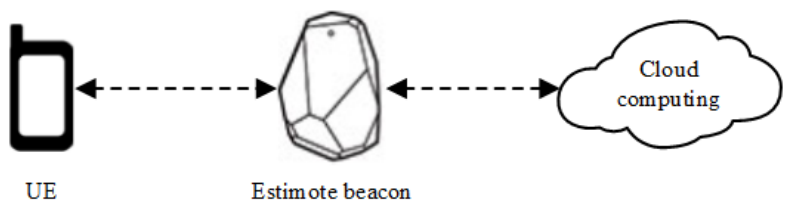

Fig. 11. Architecture of Estimote

Combined with an application for smart UE (smartphones, tablets), they provide customers with information about the store's business profile, current offers and discounts. On the other hand, the owner can find out which products are the most popular. Estimote beacons are based on Bluetooth 4.0 and provide coverage within a radius of up to $70 \mathrm{~m}$ [23].

\section{CONCLUSIONS}

Most positioning systems used for navigation purposes rely on GPS location technology, which is now applied in a wide variety of products. However, they can function accurately only in open environments, with no or few obstacles, where LOS conditions are provided to at least four satellites. Nowadays, there exists a growing demand for localization services in order to provide users with information at any time and everywhere.

It can be noticed, that RSS-based navigation systems are becoming considerably more popular amongst other positioning techniques. In this case signal strength information about all wireless devices is available at no extra cost. The benefit of choosing IEEE 802.11 technology is the fact, that deployment of an ad hoc infrastructure can be avoided since the same equipment can be used for both network access and localization purposes. Of course, hybrid location algorithms including e.g. GPS, Bluetooth, RFID or cellular network signals can raise localization accuracy. On the other hand, extending the number of channels used puts more stress on the positioning device, since it has a wider range of frequency to scan.

Positioning techniques can be used for a large number of purposes, including navigation inside buildings such as airports, museums, conference centers and other venues. Alike classic audio guides, the system may provide with additional information about different POI (Point Of Interest). Positioning systems can detect the presence of people, monitor user traffic and determine movement patterns for various security purposes. They are used by shopping centers to analyze customer behavior in order to notify about discounts and special offers, also optimize store infrastructure and improve marketing effectiveness.

Due to the rapid evolution of mobile computing, indoor positioning could make sorting and storing goods in warehouses much easier, simply by applying RFID tags. Together with sensors build in handheld devices, new platforms could be developed in the field of industrial plants, real estate management, or even mining and drilling industry. Furthermore, thanks to the availability and widespread of smartphones, LBS services will certainly help overcome language barriers and make the life of people with disabilities much easier.

\section{REFERENCES}

[1] K. W. Kolodziej, J. Hjelm, Local Positioning Systems: LBS Applications and Services, CRC Press, 2006.

[2] A. Küpper, Location Based Services - Fundamentals and Operation, John Wiley \& Sons, 2005.

[3] H. Liu, H. Darabi, P. Banerjee, J. Liu, "Survey of Wireless Indoor Positioning Techniques and Systems”, IEEE Transactions on Systems, Man, and Cybernetics, vol. 37, no. 6, pp. 1067-1080, 2007.

[4] A. Schmidt-Dannert, "Positioning Technologies and Mechanisms for Mobile Devices”, TU-Berlin, pp. 1-11, 2010.

[5] Y. Gu, A. Lo, I. Niemegeers, "A Survey of Indoor Positioning Systems for Wireless Personal Networks”, IEEE Communications Surveys \& Tutorials, vol. 11, no. 1, pp. 13-32, 2009.

[6] K. Kaemarungsi, P. Krishnamurthy, "Properties of Indoor Received Signal Strength for WLAN Location Fingerprinting”, MobiQuitous, pp. 1-10, 2004.

[7] M. L. Rodrigues, L. F. M. Vieira, M. F. M. Campos, "FingerprintingBased Radio Localization in Indoor Environments Using Multiple Wireless Technologies”, PIMRC, pp. 1203-1207, 2011. 
[8] P. Vorst, J. Sommer, C. Hoene, P. Schneider, C. Weiss, T. Schairer, W. Rosenstiel, A. Zell, G. Carle, "Indoor Positioning via Three Different RF Technologies”, UT, pp. 1-10, 2008.

[9] G. V. Zàruba, M. Huber, F. A. Kamangar, I. Chlamtac, "Indoor Location Tracking using RSSI Readings from a Single WiFi Access Point", Wireless Netw, vol. 13, pp. 221-235, 2007.

[10] J. Park, S. Kang, S. Kim, W. Lee, "A Study of Estimation of AP Position for Improvement of Indoor Positioning Performances”, IJCA, vol. 5, no. 2, pp. 73-80, 2012.

[11] V. Honkavirta, T. Perälä, S. Ali-Löytty, R. Piché, "A Comparative Survey on WLAN Location Fingerprinting Methods”, WPNC, pp. 243251, 2009.

[12] Smartmuseum webpage, http://www.smartmuseum.eu/index.html [access: 10.2014].

[13] IWAY webpage, http://www2.iway.nl/en/index.php/ [access: 10.2014].

[14] Walkbase webpage, http://www.walkbase.com/ [access: 10.2014].

[15] Guardly webpage, https://www.guardly.com/ [access: 10.2014].
[16] New Frontier for Google Maps Mapping. [Online]. Available: http://googleblog.blogspot.com/2011/11/new-frontier-for-google-mapsmapping.html [access: 10.2014].

[17] Skyhook webpage, http://www.skyhookwireless.com/ [access: 10.2014].

[18] Qubulus webpage, http://www.qubulus.com/ [access: 10.2014].

[19] Nokia Research Center webpage, https://research.nokia.com/ [access: 10.2014].

[20] New Alliance Helps You Find Needle in a Haystack. [Online]. Available: http://conversations.nokia.com/2012/08/23/new-alliancehelps-you-find-needle-in-a-haystack/ [access: 10.2014].

[21] HERE Delivers Precise Indoor Location Content to Qualcomm. [Online]. Available: http://www.qualcomm.com/connect/analystrelations/briefing-center/indoor-location [access: 10.2014].

[22] Qualcomm webpage, http://www.qualcomm.com/connect/analystrelations/briefing-center/indoor-location [access: 10.2014].

[23] Estimote webpage, http://estimote.com/ [access: 10.2014]. 